\title{
Interactive comment on "Atmospheric fate of a series of Methyl Saturated Alcohols (MSA): Kinetic and Mechanistic study" by Inmaculada Colmenar et al.
}

\section{Anonymous Referee \#1}

Received and published: 19 September 2019

Colmenar et al. have presented in this paper an extensive study of the atmospheric chemistry of some long-chain saturated alcohols. The manuscript contains kinetic studies involving relative rate method as well as reaction product analysis for reaction with the main atmospheric oxidants. These long-chain alcohols might have potential future use in biofuels and therefore it is essential to understand the atmospheric fate of these chemicals in advance. The material of this manuscript is relevant for publication in ACP although there are scopes for improvement in terms of presentation of data and explanation of results in certain areas of the current version of the manuscript. The quality of some data is questionable and there are several typographical errors. Therefore, I 
listed below.

Specific comments:

(i) Sec. 2.1 Kinetic experiments: The description of the experimental details for relative rate kinetic measurements involving FTIR is inadequate and some points are not clear. Is it an in situ or an ex-situ experiment? Is the White optics located inside the reaction chamber? If White absorption cell is a different cell then was there a facility for circulation of reaction mixture between the reaction chamber and the absorption cell? Are the actinic lamps located inside or outside of the reactor? I would recommend providing a schematic diagram of the whole set-up which will clarify all these issues. This would be extremely helpful for the readers to visualize and understand the whole setup.

(ii) Sec 3.1 Kinetic study: All the terms used in Table 1 should be described in this section ( $P$ 6, L 17, after the introduction of Table 1).

(iii) P 7, L 13: "the factor of hydroxyl...." - define this factor.

(iv) $P$ 7, L $23-27$ : this portion is not clear. Please explain.

(v) Sec 3.1.1 Estimation of rate coefficients: The title for this section should be modified. The method used for the estimation of rate coefficient should be mentioned in the title.

(vi) P 7, L 39: When you first introduce SAR, write its full form. Also, since a lot of discussions has been made on SAR, it would be helpful to briefly describe the basics of SAR method in this section.

(vii) P 9, L 27-28: All the IR bands mentioned here are not labeled in Fig. S2. Also, the font size for the labels is too small.

(viii) P 10, L 8: "It should be noted that these data should be taken with caution, since they could imply many sources of error" - Please discuss all possible sources of error.

(ix) P 10, L 21-24: The large difference between the yields of E-4-methylcyclohexanone obtained using the SPME/GC-TOFMS and FTIR is surprising. The authors argued that 
the difference in reactor volume could be the reason behind. This is not clear to me. Please explain in details.

(x) Table 1: The terms used in the table are not described either in the main text or in the legend of the table. What are the quantities listed in column 4 and 5 ? The values listed in Column 4 appear to be average of the values presented in Column 3, yet the same notation for the two columns was used. The uncertainties for some values are extremely high (sometimes close to $50 \%$ !!) which is unacceptable. A detailed discussion on the possible sources and high values of the uncertainties should be presented in the text.

(xi) Table 6: Total $\mathrm{C}$ balance for some reactions (particularly for $\mathrm{Cl}$ reactions) is extremely low. Please explain.

Technical issues:

The language in some parts of the manuscript could be improved. l've noticed some typos and other technical issues throughout the manuscript which are listed below. I have not thoroughly checked for technical issues in supplementary material and l'd request the authors to review this section again.

(i) Title: Some words of the title are written in title case while other words are not. Consistency should be maintained.

(ii) Abstract: P1 L13 - is the full stop at the end of this line valid? It looks like the sentence is continuing in the next line. P1, L20: when you first introduce $\mathrm{HCOH}$, write its full chemical name.

(iii) P2, L25: Change "Others" to "Other".

(iv) P3, L23: the rate coefficient for the reaction with MSA is termed as kS here while in equation (1) it is termed as kMSA. Please, correct. Also, define kS and kR here.

(v) P4, L5: equation (1) is written in Roman (I). Please change. 
(vi) P4, L5: 1st and 2nd brackets are wrongly placed for both the terms.

(vii) In many places, hydrogen "subtraction" is written instead of "abstraction".

(viii) P6, L30: add "for MSA" after “. . NO3 are higher".

(ix) P7, L2: write the full word "molecule", not the abbreviation "molec" in the unit.

(x) P7, L10: change "not" to "no".

(xi) P8, L1: "develop" can be changed to "developed".

(xii) P8, L19-20: check Units.

(xiii) P8, L23-24: These values could be included in Table 2.

(xiv) P8, L36: "Bands that are due...." - sentence is incomplete.

(xv) P9, L15: "of Fig. 3" can be changed to "in Fig. 3".

(xvi) P11, L4 (and in other places): "stablish" can be replaced by "establish".

(xvii) P14, L22: change "MSA have not a" to "MSA do not have a".

(xviii) Table 2: "Ratio" - "R" is capital in one place and small in the other two places.

(xviv) Fig.1 B): Left axis - correct problem with 1st and 3rd brackets.

(xvv) Fig2. Title: change "4-methylcyclohexanona" to "4-methylcyclohexanone".

(xvvi) Fig3. Picture quality is poor. Axis fonts are not readable.

(xvvii) Fig.4: the unit of $x$-axis missing. Describe the terms used in both the axis.

(xviii) Fig7 \& 8: the dot sign of radical is missing in some places. 